\title{
Designing Green Wireless Access Networks: Optimizing towards Power Consumption versus Exposure of Human Beings
}

\author{
Margot Deruyck, Wout Joseph, Emmeric Tanghe, David Plets, and Luc Martens \\ Ghent University/iMinds, Dept. of Information Technology \\ Gaston Crommenlaan 8 box 201, B-9050 Ghent, Belgium \\ Telephone: +32933149 08, Fax: +3293314899 \\ Email: margot.deruyck@intec.ugent.be
}

\begin{abstract}
When developing future networks, both power consumption and exposure of human beings will become important parameters. Here, a capacity based deployment tool is proposed whereby the developed network is optimized towards power consumption, power consumption while satisfying a certain exposure limit, or exposure accounting for coverage and capacity requirements. Applying this tool on a realistic suburban area in Ghent, Belgium for LTE-Advanced shows that power consumption and exposure optimizations contradict and that a compromise between both is required.
\end{abstract}

\section{INTRODUCTION}

The last few years, the worldwide use of mobile devices has grown considerably. Laptops, tablets, smartphones, etc. have entered into our daily life. Due to this growth, wireless access networks have also been expanding significantly in order to serve all mobile users. However, wireless access networks, and more specifically the base stations of these networks, are currently already very large power consumers [1]. As it is expected that the amount of mobile devices and customers will further increase in the coming years, it is very important to develop and implement energy-efficient wireless access networks in the near future [1], [2], [3]. In addition to this, people are becoming more and more concerned about the health effects that can be caused by these networks, so on the other side, it is also important to minimize the exposure of human beings to these networks. In this study, a capacity based deployment tool for future green wireless access networks is proposed. 'Capacity based' means that the designed network will respond to the actual bit rate requirements from the users in the network. Besides this, the tool will also optimize the network towards power consumption or towards minimizing exposure to human beings. We will apply the tool on a realistic outdoor case on a suburban area in Ghent, Belgium. This allows us to compare the effects on the network of minimizing the power consumption and the exposure of human beings.

\section{TOOL FOR DESIGNING GREEN WIRELESS ACCESS NETWORKS BASED ON REQUIRED USER CAPACITY}

In this section, the algorithm used in the deployment tool is discussed. First, we discuss how realistic traffic is generated because this is an important parameter as the developed networks will be capacity based as mentioned above. Second, the approach to minimize the power consumption is discussed. Third, the approach to minimize the power consumption of the network while satisfying a certain exposure limit is explained and finally, the approach to minimize the exposure of human beings is discussed.

\section{A. Generating realistic traffic}

The output of this step will be a collection of files, containing information about the number of users active in the network, the location of each user and the bit rate that each user requires [4]. For each time stamp, one file is generated. The following distributions are used:

- User distribution: the user distribution determines the maximum number of users that are simultaneously active for a certain time interval. This depends of course on the considered area and the population density of this area. The data here used is obtained by processing measurements [5] and is confirmed by [6], [7]. As we assume the maximum number of simultaneously active users, no arrival rate of users is defined as the worst case scenario is considered.

- Location distribution: determines the location of each individual user within the considered area. Here, a uniform distribution is considered whereby the users are uniformly spread over the area of interest resulting in equidistant discrete samples both in $\mathrm{x}$ and $\mathrm{y}$ direction.

- Bit rate distribution: determines the bit rate that is required by each user individually. There will be users requiring $64 \mathrm{kbps}$ (i.e., making a phone call) and users requiring $1 \mathrm{Mbps}$ (i.e., using data services). These assumptions are made based on private interviews with Belgian operators.

By combining all the distributions above, it is possible to provide for each time stamp a file with the number of users that are active and for each user the location and the bit rate he/she requires. These files are used as input for the tool. Note that all the distributions here considered are assumed to be independent of each other. 


\section{B. Minimizing the power consumption}

In Fig. 1, the algorithm for minimizing the power consumption is presented. As input, the traffic files developed in the previous section (Section II-A) are used [4]. Furthermore, a file is provided with the locations of the base stations of the network that needs to be optimized. Note that no base stations will be added to the network. The only possible operation is to put base stations into sleep mode or to wake them up. Also a shape file of the considered area is provided.

For each time stamp, a network will be developed. Due to the different distributions used to generate the traffic, multiple simulations are needed per time stamp to develop the network for that time stamp. Here, 40 simulations are necessary to obtain a good estimation of the expected value of the different parameters (power consumption, exposure, etc.). Note, that this number only applies on the selected scenario. The algorithm will thus be repeated $n \cdot s$ times with $n$ the number of time stamps and $s$ the number of simulations (Step 1 in Fig. 1).

For each time stamp, we start with all the base stations in sleep mode. Next, we try to find an appropriate base station to connect the users with (Step 2). In terms of power consumption, it is more energy-efficient to connect a user with an active base station than waking up a base station as increasing the antenna's input power results in an electric power increase of up to $96 \mathrm{~W}$ while waking a base station up results in an additional electric power of $920 \mathrm{~W}$ which is a factor 10 higher (assuming that a base station consumes $45 \%$ of its power while in sleep mode [9]). Therefore, the active base stations are sorted according to the path loss experienced by the user (Step 3). To determine this path loss, the following procedure is followed. Firstly, the direct line from the user to the base station is determined. Secondly, it is checked if there is any building obstructing this direct line. If so, the Walfisch-Ikegami (WI) non-Line-of-Sight (nLoS) model is used, otherwise the WI Line-of-Sight (LoS) model [10]. Furthermore, each user is assigned a certain shadowing margin. To determine this margin, a normal distribution is used with $\sigma$ equal to the standard deviation of the propagation model i.e., WI. Next, the algorithm runs over the ordered list of base stations (Step 4). The user is assigned to the first base station that can offer the bit rate required to the user (Step 5). There are two criteria that have to be checked: (i) is the path loss experienced by the user lower than the maximum allowable path loss (i.e., the path loss to which a transmitted signal can be subjected while still being detectable at the receiver)? and (ii) has the base station still enough capacity available to provide this required bit rate? If the answer to the last criterion is negative, the base station can always try to switch to a higher modulation or coding rate. However, this can only be done when the users already assigned to this base station can still be served (path loss and bit rate). If there are no active base stations or in the case that none of the active base stations can serve the user, an inactive base station should be woken up. The same procedure as above is followed: all inactive base stations are sorted according to the path loss experienced by the user (Step 6), we run over the sorted list (Step 7), and the user is assigned to the first base station that can serve the user (Step 8). This base station will thus be activated (Step 9). If an inactive base station is woken up, it is useful to check if there are users that might experience a lower path loss from this 'new' base station (Step 10). If none of the inactive base stations can serve the user or if all the base stations are active, it is concluded that the user can not be served (Step 11). To determine the power consumption of the base station, the model in [1] is used. When the base station is in sleep mode, it is assumed that its power consumption is reduced with $45 \%$ [9]. For the first time stamp $(i=1)$, all base stations are asleep.

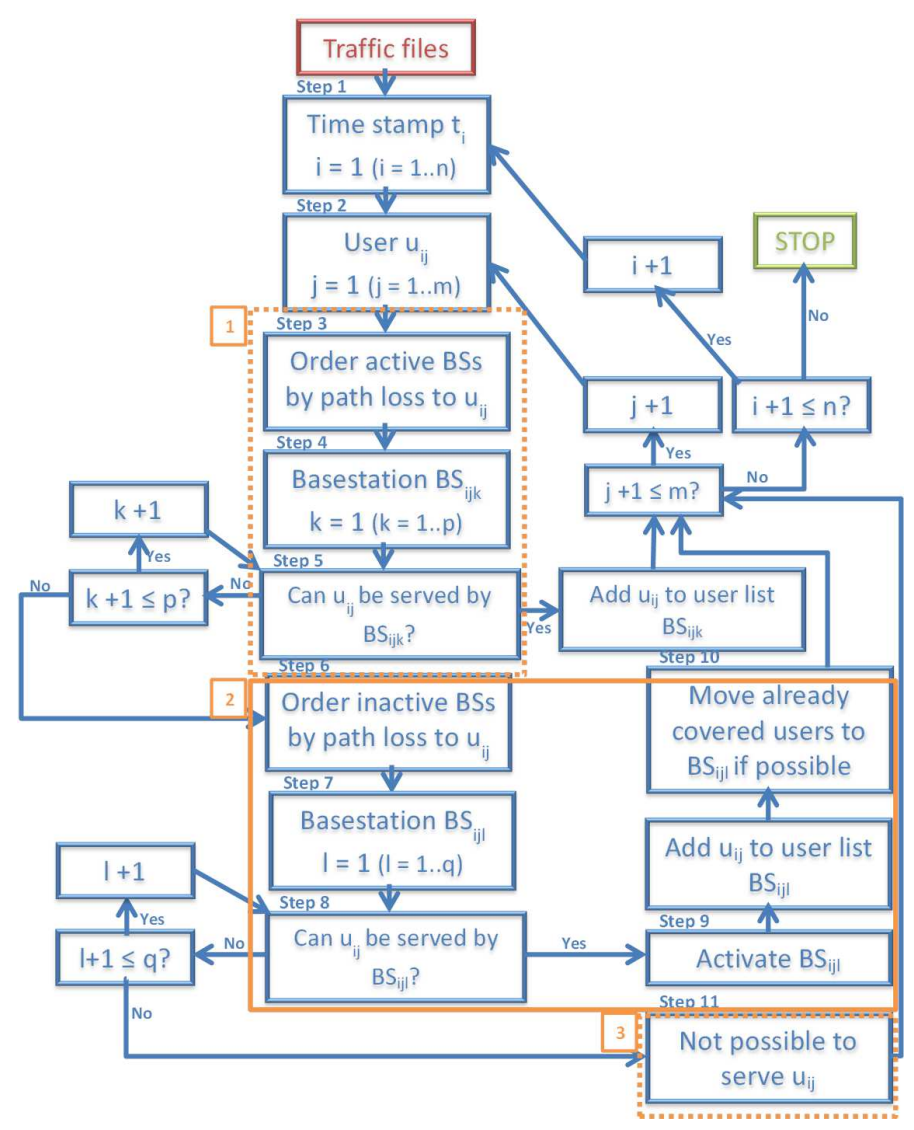

Fig. 1. Flow diagram of the capacity based deployment tool for designing energy-efficient wireless access networks with a minimal power consumption.

\section{Minimizing the power consumption while satisfying a cer- tain exposure limit}

The algorithm here described minimizes the power consumption while satisfying a certain exposure limit per antenna. The same procedure as in Section II-B is used, however, an extra step will be added in the beginning of the algorithm. In this step, we determine for each base station the maximum EIRP (Equivalent Isotropically Radiated Power) to satisfy the considered exposure limit per antenna [11]. Once this maximum EIRP is known, the maximum input power of the antenna can be calculated. The network can then be developed by using the procedure described in Section II-B with the 
obtained limitations for the antenna input power of each base station.

\section{Minimizing the exposure of human beings}

Fig. 2 shows the flow diagram of the approach followed to design wireless access networks with a minimal exposure for human beings which is based on the algorithm proposed in [11].

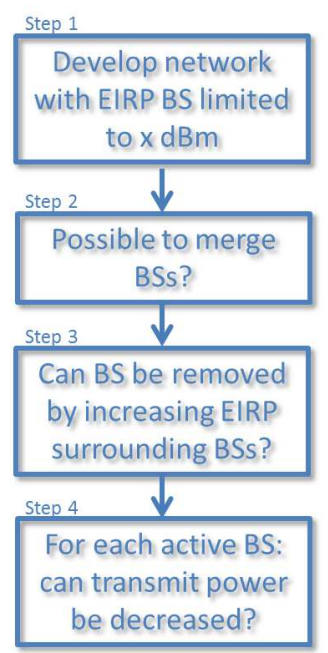

Fig. 2. Flow diagram of the capacity based deployment tool for designing wireless access networks with a minimal exposure of human beings.

In the first step (Fig. 2 Step 1), the network will be created whereby the EIRP (Equivalent Isotropically Radiated Power) of the base stations is limited to $x \mathrm{dBm}$. In this way, a network containing many low-power base stations is obtained which is, in terms of minimal exposure, preferred over a network with a few high-power base stations. The algorithm discussed in Section II-B can be used in this step.

In the second step (Fig. 2 Step 2), it is investigated if two base stations can be merged into one base station with whether or not a higher transmit power. Only those pairs of base stations are investigated whereby the base stations are laying in $125 \%$ of their range from each other [11]. This value gives us a good trade-off between not excluding possibly mergeable base stations and not needlessly investigating all base station pairs. To determine the location of the new base station, a list of users that are not covered any more when removing the base station pair is composed. These users need to be covered by a new base station with an as low as possible transmit power. To this end, the algorithm determines for each possible base station location the highest path loss obtained between that location and the uncovered users. The base station location that has the lowest value (and can provide the required capacity) will be able to cover the users with the lowest possible transmit power and will thus be added as new base station to the network only when the global exposure of the network $E_{M}$, which is a weighted average of the median electric-field strength $E_{50}$ and the $95 \%$-percentile value of the field strengths $E_{95}$, is lower than before the merge [11]:

$$
E_{M}=\frac{w_{1} \cdot E_{50}+w_{2} \cdot E_{95}}{w_{1}+w_{2}}
$$

with $w_{1}$ and $w_{2}$ weighting factors for the 50\%-percentile value $E_{50}$ and the $95 \%$-percentile value $E_{95}$ respectively. In this way, the median exposure of the network $\left(E_{50}\right)$ and the maximum exposure values of the network $\left(E_{95}\right)$ are taken into account [11]. Here, we assume an equal impact of $E_{50}$ and $E_{95}$ on the metric and set both $w_{1}$ and $w_{2}$ at a value of 0.5 . The third step (Fig. 2 Step 3) investigates if it is possible to remove a base station by increasing the transmit power of the surrounding base stations. The algorithm starts with the base station covering the lowest number of users because this has the highest chance to cover the users by other base stations. All users that are covered by the base station under investigation need to be covered by another base station. Therefore, the transmit power of a first base station is increased until all users are covered or until it has reached its maximum capacity or until the maximum allowable transmit power is reached. When there are still uncovered users, the transmit power of the next surrounding base station is increased. A base station that has already increased its transmit power will not be considered any more in further investigations. The adaptation of the network will only be accepted when the global exposure of the network is lower than before.

In the fourth and last step (Fig. 2 Step 4), it is checked if the transmit power of each base station can be decreased while still serving the users.

\section{SELECTED SCENARIO}

The algorithms described above will be applied on a realistic case for an outdoor suburban area in Ghent, Belgium. This area of $6.85 \mathrm{~km}^{2}$ is shown in Fig. 3. We only considered the time stamp of the day with the maximum number of users i.e., 224 users (green circles in Fig. 3), so the worst-case scenario is investigated. 75 base station locations are considered (blue triangles in Fig. 3). These are the locations of existing base stations of three different Belgian operators. It is assumed that the operators share their network and thus each user can connect to each base station. Each of the base stations can be switched on or put into sleep mode. Furthermore, LTEAdvanced is used as wireless technology with a frequency of $2.6 \mathrm{GHz}$ and a bandwidth of $5 \mathrm{MHz}$ with only one transmitting and receiving antenna (i.e., SISO (Single Input Single Output). The other link budget parameters are assumed to be the same as in [12].

For evaluating the exposure, we use a grid with a distance of $50 \mathrm{~m}$ between two different grid points in both horizontal as vertical direction (which is a different grid from the one in Fig. 3). This results in a total of 2737 grid points. The exposure of a certain base station will not be taken into account for the grid points within a distance of $25 \mathrm{~m}$ from that specific base station. To determine the electic-field strength of a single antenna and the combined electric-field strength from multiple sources, the approach of [11] is followed.

For the scenario described above, three different networks 
will be developed: one optimized towards power consumption (Section II-B), one optimized towards power consumption satisfying an electric field of $4.48 \mathrm{~V} / \mathrm{m}$ per antenna at $2.6 \mathrm{GHz}$ (mandated by the Flemish exposure standard [13], Section II-C), and one optimized towards minimal global exposure (Section II-D, Eq. (1)). 40 simulations will be needed per considered optimization. For the optimization towards exposure, the maximum input power of the antenna is limited to $35 \mathrm{dBm}$ as mentioned above.

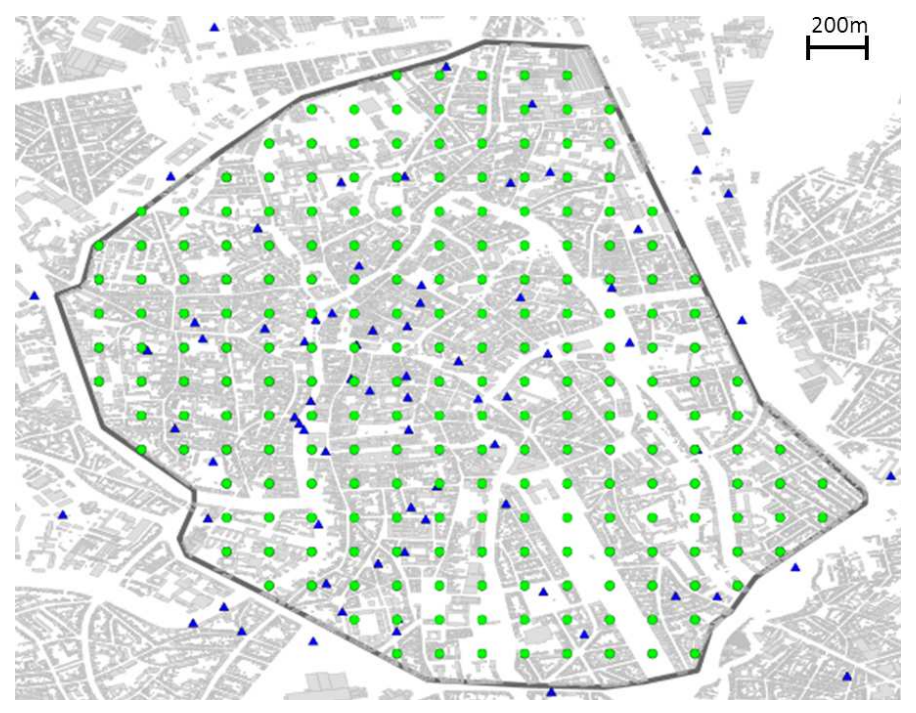

Fig. 3. The selected suburban area of $6.85 \mathrm{~km}^{2}$ in Ghent, Belgium. A green circle indicates a user and a blue triangle a possible base station location.

\section{RESULTS}

Table I gives an overview of the different performance parameters (number of active base stations, obtained electricfield strength, power consumption of the network (active and sleeping base stations), and percentage of users served) for the three different optimizations. For each parameter, the 50th percentile calculated over the 40 simulations is shown. For the obtained electric-field strength (i.e., exposure value in $\mathrm{V} / \mathrm{m}$ ), we also added the maximum exposure value (95th percentile). The probability density function of the obtained field exposure is shown in Fig. 4.

As expected, the lowest power consumption in Table I is of course obtained when optimizing towards this parameter. The power consumption amounts to $66.1 \mathrm{~kW}$, while for the other two optimizations a power consumption of about $67.0 \mathrm{~kW}$ is found. This lower power consumption is because the network uses only 16 active base stations, while for the other two cases, the network requires even up to 23 active base stations. Due to this low number of active base stations, a higher input power of the base station's antenna will be necessary to cover the users. This higher input power results unfortunately in the highest exposure of the considered optimizations (Fig. 4 and Table I). An average value of $75 \mathrm{mV} / \mathrm{m}$ and a maximum of $213 \mathrm{mV} / \mathrm{m}$ is obtained.

The lowest field exposure $(30 \mathrm{mV} / \mathrm{m}$ on average and $89 \mathrm{mV} / \mathrm{m}$ maximum) is obtained, as expected, when optimizing towards global exposure. To attain this low exposure, a low antenna's input power per base station is used, resulting in a total number of 23 active base stations. This leads to a slightly higher power consumption i.e., $67.3 \mathrm{~kW}$, than when optimizing towards power consumption. Note however, that the percentage of covered users is significantly lower for the network optimized towards exposure than for the network optimized towards power consumption (96.4\% versus $99.1 \%$ ) which explains why the power consumption is only slightly higher when optimizing towards exposure than towards power consumption. This lower coverage is due to the limitation of the antenna's input power when optimizing towards exposure (Section II-D).

A compromise between optimizing towards power consumption and exposure is optimizing towards power consumption while satisfying an exposure limit. As shown in Fig. 4, the field exposure of this network $(67 \mathrm{mV} / \mathrm{m}$ on average and $197 \mathrm{mV} / \mathrm{m}$ maximum) is between the field value of the networks optimized towards power consumption and global exposure. The field strength obtained when optimizing towards global exposure is however 2 times lower than when optimizing towards power consumption while satisfying a certain exposure limit. The same is true for the number of active base stations (19 base stations) and thus the power consumption $(67.0 \mathrm{~kW})$. Also the percentage of covered users $(98.9 \%)$ is in between the results for the networks optimized towards power consumption and exposure. This is due to the fact that the network is optimized towards power consumption, but still takes the exposure into account (by limiting the input power of the antennas). Optimizing the network towards power consumption while satisfying a certain exposure limit is thus a comprise between optimizing towards power consumption and global exposure but field values are a factor 2 higher.

\begin{tabular}{|c|c|c|c|c|c|}
\hline \multirow[t]{2}{*}{ Optimized towards } & \multirow[t]{2}{*}{$\begin{array}{l}\text { No. of active base stations } \\
{[-]}\end{array}$} & \multirow[t]{2}{*}{$\begin{array}{c}\text { Power consumption } \\
{[\mathrm{kW}]}\end{array}$} & \multicolumn{2}{|c|}{$\begin{array}{c}\text { Exposure } \\
{[\mathrm{mV} / \mathrm{m}]}\end{array}$} & \multirow[t]{2}{*}{$\begin{array}{c}\text { Users served } \\
{[\%]}\end{array}$} \\
\hline & & & $\mathrm{p}_{50}$ & $\mathrm{p}_{95}$ & \\
\hline Power consumption & 16 & 66.1 & 75 & 213 & 99.1 \\
\hline $\begin{array}{l}\text { Power consumption } \\
\text { satisfying } 4.48 \mathrm{~V} / \mathrm{m}\end{array}$ & 19 & 67.0 & 67 & 197 & 98.9 \\
\hline Exposure & 23 & 67.3 & 30 & 89 & 96.4 \\
\hline
\end{tabular}

TABLE 1. OVERVIEW OF THE PERFORMANCE OF THE DEVELOPED NETWORK (40 SIMULATIONS) WHEN OPTIMIZING TOWARDS POWER CONSUMPTION, POWER CONSUMPTION SATISFYING $4.48 \mathrm{~V} / \mathrm{M}$, AND EXPOSURE.

\section{CONCLUSiON}

In this study, a capacity based deployment tool for future green wireless access networks is proposed. 'Capacity based' means that the designed network will respond to the actual bit rate requirements from the users in the area. Three different algorithms are proposed for optimizing the network towards power consumption, power consumption while satisfying a certain exposure limit, and minimal global exposure. These algorithms are applied on a realistic suburban area in Ghent, Belgium for LTE-Advanced.

The results show that when optimizing towards power consumption results in a lower power consumption $(66.1 \mathrm{~kW})$ but in higher exposure values (213 $\mathrm{mV} / \mathrm{m}$ maximum), while optimizing towards exposure results in a lower exposure $(89 \mathrm{mV} / \mathrm{m}$ 


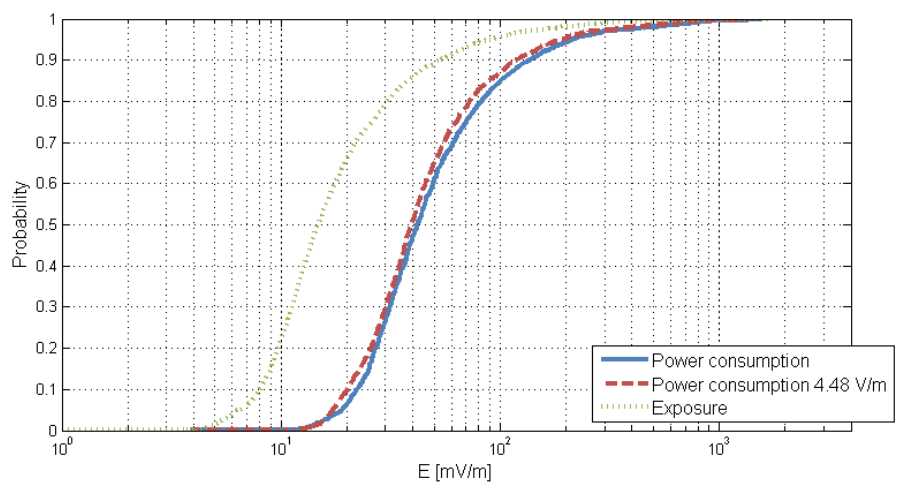

Fig. 4. Probability density function of the obtained exposure when optimizing towards (i) power consumption, (ii) power consumption satisfying $4.48 \mathrm{~V} / \mathrm{m}$, and (iii) global exposure.

maximum) but a higher power consumption $(67.3 \mathrm{~kW})$. When optimizing towards power consumption while satisfying a certain exposure limit, a compromise between optimizing towards power consumption and field is found. The obtained power consumption $(67.0 \mathrm{~kW})$ and exposure $(197 \mathrm{mV} / \mathrm{m}$ maximum $)$ are in between the results of the other optimizations.

\section{REFERENCES}

[1] M. Deruyck, W. Vereecken, W. Joseph, B. Lannoo, M. Pickavet, L. Martens, Reducing the power consumption in wireless access networks: overview and recommendations, Progress In Electromagnetics Research, Vol. 132, 2012, pp. 255-274.

[2] M.A. Marsan, L. Chiaraviglio, D. Ciullo, M. Meo, A Simple Analytical Model for the Energy-Efficient Activation of Access Points in Dense WLANs, Proceedings of the 1st International Conference on EnergyEfficient Computing and Networking (e-Energy), 2010, pp. 159-168.

[3] G. Koutitas, Green Network Planning of Single Frequency Networks, IEEE Transactions on Broadcasting, Vol. 56, No. 4, December 2010, pp. 541-550.

[4] M. Deruyck, W. Joseph, E. Tanghe, L. Martens, Reducing the Power Consumption in LTE-Advanced Wireless Access Networks by a Capacity Based Deployment Tool, Radio Science, Minor revision, 2014.

[5] M. Deruyck, E. Tanghe, W. Joseph, L. Martens, Characterization and optimization of the power consumption in wireless access networks by taking daily traffic variations into account, Eurasip Journal on Wireless Communication and Networking, 2012, doi: 10.1186/1687-1499-2012248.

[6] W. Joseph, L. Verloock, Influence of mobile phone traffic on general public base station exposure, Health Physics, Vol. 99, No. 5, 2010, pp. 631-638.

[7] A. Gati, A. Hadjem, M.F. Wong, J. Wiart, Exposure Induced by WCDMA Mobile Phones in Operating, IEEE Transactions on Networks, Vol. 8, No. 12, 2009, pp. 5723-5727.

[8] G. Auer, O. Blume, V. Giannini, I. Godor, M.A. Imran, Y. Jading, E. Katranaras, M. Olsson, D. Sabella, P. Skillermark, W. Wajda, EARTH Deliverable D2.3: Energy efficiency analysis of the reference systems, areas of improvements and target breakdown, January 2013.

[9] M.J. Gonzalez, D. Ferling, W. Wajda, A. Erdem, P. Maugars, Concepts for Energy Efficient LTE Transceiver Systems in Macro Base Stations, Future Network \& Mobile Summit, 2011, ISBN: 978-1-905824-25-0.

[10] COST Action 231, Digital mobile radio towards future generation systems, Chapter 4, ISBN: 92-828-5416-7.
[11] D. Plets, W. Joseph, K. Vanhecke, L. Martens, Exposure Optimization in Indoor Wireless Networks by Heuristic Network Planning, Progress In Electromagnetics Research, Vol. 139, 2013, pp. 445-487.

[12] M. Deruyck, W. Joseph, L. Martens, Power Consumption Model for Macrocell and Microcell Base Stations, Transactions on Emerging Telecommunications Technologies, doi: 10.1002/ett.2565.

[13] Besluit van de Vlaamse Regering van 19 november 2010 tot wijziging van het besluit van de Vlaamse Regering van 1 juni 1995 houdende algemene en sectorale bepalingen inzake milieuhygiëne wat betreft de normering van vast en tijdelijk opgestelde zendantennes voor elektromagnetische golven tussen $10 \mathrm{MHz}$ en $10 \mathrm{GHz}$. 